\title{
Relación entre asertividad, rendimiento académico y ansiedad en una muestra de estudiantes mexicanos de secundaria
}

\author{
Claudia González Fragoso ${ }^{1 *}$, Yolanda Guevara Benítez², David Jiménez Rodríguez ${ }^{1}$ y Raúl J. Alcázar Olán ${ }^{3}$ \\ ${ }^{1}$ Universidad Autónoma del Estado de Hidalgo, México; ${ }^{2}$ Universidad Nacional Autónoma de México, México; \\ ${ }^{3}$ Universidad Iberoamericana Puebla, México
}

\author{
Recibido, febrero 16/2017 \\ Concepto de evaluación, abril 28/2017 \\ Aceptado, julio 15/2017
}

\begin{abstract}
Referencia: González Fragoso, C., Guevara Benitez, Y., Jiménez Rodríguez, D. \& Alcázar Olán, R.J. (2018). Relación entre Asertividad, Rendimiento Académico y Ansiedad en una Muestra de Estudiantes Mexicanos de Secundaria. Acta colombiana de Psicología, 21(1), 116-127. doi: http://www.dx.doi.org/10.14718/ACP.2018.21.1.6
\end{abstract}

Resumen

\begin{abstract}
Las habilidades sociales, en su forma más avanzada, están dirigidas a resolver problemas inmediatos de la situación, a la vez que minimizan la probabilidad de futuros problemas. Específicamente, la habilidad reconocida como asertividad puede guardar una relación con el rendimiento académico y el nivel de ansiedad en adolescentes. Teniendo esto en cuenta, el objetivo de la presente investigación es conocer la relación entre el nivel de asertividad, el rendimiento académico y diversos componentes de la ansiedad en una muestra de estudiantes mexicanos de secundaria, así como explorar posibles diferencias por sexo. Para esto, se hace un análisis descriptivo y correlacional, donde se utilizan dos instrumentos: la Escala de Conducta Asertiva para Niños y la Escala de Ansiedad Manifiesta en Niños (revisada); además del promedio de calificaciones como indicador del rendimiento académico. Se utilizó una muestra de 535 adolescentes (54.6\% mujeres y $45.4 \%$ hombres), con un promedio de edad de 13.01 años $(D E=.74$, rango de 11 a 17 años). Se realizó un muestreo por conglomerados y conveniencia, donde se abarcó la totalidad de los alumnos de los dos primeros grados escolares de una escuela pública del Estado de Hidalgo. Los resultados indican diferencias por sexo en todas las variables de estudio y correlaciones estadísticamente significativas entre asertividad y ansiedad $(r=.182, p<.01)$, entre rendimiento académico y asertividad $(r=-.203, p<.01)$, y entre el rendimiento académico y el componente preocupaciones sociales de la escala de ansiedad $(r=-.124, p<.01)$, aunque estas últimas fueron de baja magnitud.

Palabras clave: Habilidades sociales, asertividad, rendimiento académico, ansiedad, estudiantes de secundaria.
\end{abstract}

\section{Relationship between Assertiveness, Academic Performance and Anxiety in a Sample of Mexican Students in Secondary Education}

\begin{abstract}
Social skills in their most advanced form are aimed at solving any immediate problems of a situation while minimizing the likelihood of future problems. This is also known as assertiveness, a variable that can be related to academic performance and the level of anxiety in adolescents. This research is descriptive and correlational with the aim of knowing the relationship between level of assertiveness, academic performance and various components of anxiety in a sample of Mexican high school or secondary students, as well as exploring possible differences related to sex. This study uses two instruments: Children's Assertive Behavior Scale (CABS) and the Revised Children's Manifest Anxiety Scale ${ }^{\mathrm{TM}}$. The grade point average (GPA) was taken as an indicator of academic performance. The participants were 535 adolescents ( $54.6 \%$ female, $45.4 \%$ male), with a mean age of 13.01 years ( $s t d=.74$, range 11 to 17 years). Cluster sampling and convenience sampling were used, encompassing all students in the first two grades of a public or state school in the state of Hidalgo. The results indicate sex differences in all study variables. We also found statistically significant correlations between assertiveness and anxiety $(r=.182, p<.01)$, between academic performance and assertiveness $(r=-.203, p<.01)$, and between academic performance and the social concerns component of the anxiety scale $(r=-.124, p<.01)$, although these were low in magnitude.

Key words: Social skills, assertiveness, academic performance, anxiety, high school students
\end{abstract}

\footnotetext{
* Instituto Ciencias de la Salud, Universidad Autónoma del Estado de Hidalgo. Circuito Ex-Hacienda de la Concepción s/n, Carretera Pachuca - Tilcuautla, C.P. 42060, Hidalgo, México. Tel: (0052) 7717172000 Ext. 5104, claudiaglezf@gmail.com
} 


\title{
Relação entre Assertividade, Desempenho Acadêmico e Ansiedade numa Amostra de Estudantes Mexicanos de Secundária
}

\begin{abstract}
Resumo
As habilidades sociais, em sua forma mais avançada, estão voltadas a resolver problemas imediatos da situação enquanto diminuem a probabilidade de futuros problemas. Isso também é conhecido pelo termo assertividade, variável que pode estabelecer relação com o desempenho acadêmico e com o nível de ansiedade em adolescentes. Esta pesquisa foi descritiva e correlacional, teve como objetivo conhecer a relação entre o nível de assertividade, o desempenho acadêmico e diversos componentes da ansiedade numa amostra de estudantes mexicanos de secundária (equivalente ao ensino médio no Brasil), bem como explorar possíveis diferenças por sexo. Utilizaram-se dois instrumentos: Escala de Comportamento Assertivo para Crianças e Escala de Ansiedade Manifestada em Crianças (revisada); a média de qualificações foi tomada como indicador de desempenho acadêmico. A amostra foi composta por 535 adolescentes (54.6\% mulheres, $45.4 \%$ homens), com uma média de idade de 13.01 anos $(D P=.74$, na faixa dos 11 aos 17 anos). A amostra foi por conglomerados e conveniência, abrangendo a totalidade dos estudantes dos primeiros anos escolares de uma escola pública do estado de Hidalgo. Os resultados indicam diferenças por sexo em todas as variáveis de estudo. Também se encontraram correlações estatisticamente significativas: entre assertividade e ansiedade $(r=.182, p<.01)$, entre desempenho acadêmico e assertividade $(r=-.203, p<.01)$, e entre o desempenho acadêmico e o componente preocupações sociais da escala de ansiedade $(r=-.124, p<.01)$, embora estas tenham sido de baixa magnitude.

Palavras-chave: Ansiedade, assertividade, desempenho acadêmico, estudantes de secundária, habilidades sociais.
\end{abstract}

\section{INTRODUCCIÓN}

Desde la psicología cognitivo-conductual, el término competencia social involucra tres componentes: el socioafectivo, que incluye aspectos como apego, expresividad y autocontrol; el sociocognitivo, que abarca conocimiento social, toma de una perspectiva, atribuciones y razonamiento moral; y la conducta social, que incluye habilidades de comunicación verbal y no verbal, cooperación, apoyo, participación y manejo de conflictos. De este modo, la competencia social implica habilidades y conocimientos necesarios para desenvolverse con éxito en las distintas interacciones sociales (Caballo, 1993).

En lo que se refiere específicamente a las habilidades sociales, Ribes, Bisquerra, Agulló, Fililla y Soldevila (2005) las definen como la capacidad de establecer relaciones sociales satisfactorias; lo que implica reconocer las emociones de los demás, ayudar a otras personas a sentirse bien, desarrollar la empatía, ser asertivo y solucionar conflictos.

Por otra parte, la teoría del aprendizaje social, iniciada por Bandura (1982), sirve como base para explicar el desarrollo de dicha conducta. Desde este marco, las habilidades sociales se adquieren por lo general como consecuencia de varios mecanismos, por ejemplo: por experiencia directa, por aprendizaje vicario u observacional, por transmisión verbal o instruccional, o por feedback o retroalimentación interpersonal. Estas formas de aprendizaje operan para el desarrollo de habilidades y de expectativas cognitivas respecto a situaciones interpersonales, gracias a la acción recíproca entre las fuentes de influencia generadas por el propio individuo y aquellas que tienen origen externo.

Profundizando en el tema, el aprendizaje de las habilidades sociales inicia desde las primeras interacciones entre la madre y el bebé, durante las cuales los niños aprenden la función social de las sonrisas, los gestos y las palabras. Posteriormente, con el desarrollo de formas de comunicación más avanzadas, el niño puede incorporarse en grupos familiares y sociales cada vez más amplios. Así, las habilidades sociales se van aprendiendo y complejizando a lo largo de toda la vida, porque han de ajustarse a los requerimientos que enfrenta cada individuo en cada edad de desarrollo psicológico y en cada contexto en que se desempeña (Papalia, Olds \& Feldman, 2009). Asimismo, Bermúdez (2010) señala que dichas habilidades permiten la adaptación y el funcionamiento adecuado en el ambiente social, ya que implican un despliegue de comportamientos sociales, emocionales e intelectuales necesarios para tener un ajuste adecuado a la sociedad.

En el desarrollo de la competencia social intervienen, en estrecha interrelación, diversas habilidades conductuales -lingüísticas, motrices, de atención-, variables relacionadas con características individuales -cognitivas, afectivas y fisiológicas-, y aspectos ambientales, educativos y culturales -incluyendo los valores o normas sociales adquiridos en el hogar, el colegio y otros contextos sociales en los que se incorpora el individuo a lo largo de su vida- (García, Cabanillas, Morán \& Olaz, 2014). 
De igual forma, Monjas (2012) aclara que en la infancia las habilidades sociales incluyen todas aquellas conductas necesarias para interactuar y relacionarse con los iguales y con los adultos de forma efectiva y mutuamente satisfactoria. Específicamente, las relaciones adecuadas inician con el despliegue de habilidades básicas de interacción social, tales como sonreír, saludar, hacer favores, mostrar cortesía y amabilidad; y el establecimiento de dichas interacciones suele ser altamente reforzante para los niños y les brinda la oportunidad para desarrollar conductas sociales más complejas, tales como iniciar conversaciones, formular y responder preguntas, expresar afecto y sentimientos positivos, entre otros componentes, lo que, a su vez, les puede proporcionar mayor refuerzo social y satisfacción personal e interpersonal a corto y largo plazo (García et al., 2014).

Por otra parte, Caballo(2009) define la conducta socialmente habilidosa como el conjunto de conductas emitidas por un individuo en un contexto interpersonal, que incluye maneras adecuadas de expresar sentimientos, actitudes, deseos, opiniones o derechos de una persona, de un modo adecuado a la situación, respetando esas conductas en los demás. Dentro de este conjunto de habilidades se encuentran también las conocidas con el nombre de asertividad, las cuales constituyen uno de los aspectos más importantes a desarrollar en los niños de edad escolar, así como en adolescentes y adultos. De este modo, las habilidades sociales, en su forma más avanzada, están dirigidas a resolver problemas inmediatos de la situación, y, a la vez, minimizan la probabilidad de futuros problemas.

Asimismo, la expresión y socialización de las emociones de manera adecuada permite a los individuos desarrollar empatía y responder a las emociones de otros (Cohen, Esterkind, Lacunza, Caballero \& Martinenghi, 2011). El grado de competencia social en la infancia -y, particularmente, de asertividad - es un predictor de la adaptación escolar porque ayuda a los niños y adolescentes a adaptarse al estrés y a resolver problemas. Según Inglés, Delgado, García, Ruiz y Díaz (2010a), pueden ubicarse tres tipos de interacción mutuamente excluyentes, de acuerdo con las conductas que involucren: la interacción prosocial, agresiva o de evitación.

Para explorar el nivel de competencia alcanzado por alumnos de educación primaria y secundaria, así como el grado en que se relaciona con su rendimiento escolar y con diversas variables psicológicas, se han llevado a cabo diversos estudios, como los que se describen a continuación. Por una parte, el estudio realizado por Gutiérrez, Escartí y Pascual (2011) con 822 estudiantes con edades entre 8 y 15 años comprobó mediante modelos de ecuaciones estructurales que la conducta prosocial, la empatía y la percepción de eficacia predicen positivamente la responsabilidad personal y social de los alumnos, variables que están fuertemente asociadas con el desarrollo positivo de los jóvenes y con su desempeño en el ámbito escolar; este análisis también indicó que la agresividad mostraba relaciones negativas con la responsabilidad.

Por otra parte, Costa y Tabernero (2012) reportan que tanto el autoconcepto-académico, familiar y físico-como la edad del estudiante influyen directamente en el rendimiento escolar, cuyo indicador fue obtenido a través de las calificaciones asignadas por los profesores de diversas materias académicas en un lapso específico de tiempo. Mientras que Redondo, Inglés y García (2014), en una muestra de 2022 alumnos españoles de educación secundaria, encontraron que los chicos de segundo grado y las chicas de cuarto grado presentaban respectivamente la menor y mayor prevalencia puntual de conducta prosocial; además, pudieron comprobar que los alumnos prosociales atribuían sus éxitos académicos (altas calificaciones y altos promedios académicos en las materias de lenguaje y matemáticas) a su propio esfuerzo más que a variables externas, lo que constituye una correlación entre conducta prosocial, atribución y logro académico (rendimiento escolar). Un dato interesante de este estudio es que solo el $17 \%$ de los alumnos mostraron un patrón prosocial. Aspecto que se relaciona con el estudio de Carbonero, Martín, Monsalvo y Valdivieso (2015), pues estos reportan, de igual manera, las fuertes correlaciones que existen entre nivel de rendimiento académico, nivel de conductas prosociales y actitudes de responsabilidad social en alumnos españoles.

Igualmente, el estudio realizado por Lacunza (2009) con niños argentinos demostró que la presencia de habilidades sociales previene la aparición de comportamientos disfuncionales, en especial aquellos relacionados con la agresividad y el negativismo. Mientras que el trabajo de investigación de Oyarzún, Estrada, Pino y Oyarzún (2012) con estudiantes chilenos reporta una asociación positiva entre rendimiento escolar y comportamientos sociales adecuados en el aula.

El desarrollo social en la infancia y la adolescencia es un factor predictor del ajuste psicológico, académico y social en la etapa adulta. Y, como mencionan Mendo, León, Felipe, Polo y Palacios (2016), el grado de competencia social, además de relacionarse con el éxito personal, tiene una gran influencia en el logro de competencias laborales, incluso por encima de habilidades técnicas e intelectuales; lo que ha llevado a señalarla como una de las competencias del siglo XXI. La investigación realizada por estos autores con una muestra de jóvenes españoles indicó que el nivel de habilidades sociales no fue el óptimo; esto debido a que se obtuvieron bajas puntuaciones en las habilidades para defender sus derechos, la expresión de disconformidad, el hacer peticiones y el iniciar interacciones positivas, es decir, en los componentes de la asertividad. 
Estos resultados coinciden con los reportados por Gutiérrez y López (2015), quienes analizaron las necesidades formativas en el ámbito de las habilidades sociales en estudiantes españoles de educación secundaria. Sus datos indican que los adolescentes muestran bajos niveles de habilidades sociales, en especial en lo que respecta a las conductas asertivas, los problemas de autoconcepto y las dificultades interpersonales. Estos autores concluyen que dicha población se encuentra en continuas situaciones de riesgo al carecer de las destrezas suficientes para evitar situaciones de conflicto, lo que pone de manifiesto la importancia de enseñar habilidades socioemocionales a estos colectivos de estudiantes como una asignatura más del currículo.

Por otra parte, las personas que no han desarrollado competencia social, o asertividad, suelen tener mayores niveles de pensamientos negativos y de manifestaciones fisiológicas relacionadas con la ansiedad. Esto de acuerdo con Contreras et al. (2005), pues, según lo que reportan, la ansiedad es un estado emocional desagradable, relacionado con pensamientos negativos derivados de la evaluación de una situación percibida como amenazante; y que engloba aspectos cognitivos, fisiológicos y motores que suelen implicar conductas poco adaptativas.

De igual manera, Lacunza (2009) señala que "existe suficiente evidencia empírica que conecta las competencias sociales a la salud física y mental" (p. 233). Así, las deficiencias en las habilidades sociales se relacionan a una variedad de trastornos como la ansiedad, las enfermedades cardiovasculares y el abuso de sustancias, entre otros. En dicho estudio, realizado por diversas universidades y el Ministerio de Salud en Argentina con una amplia muestra de sujetos, la autora agrega que el $12 \%$ de los niños registraron algún tipo de problema de comportamiento social, y el $16 \%$ mostraba específicamente comportamientos vinculados a la agresividad.

En concordancia con lo anterior, investigaciones realizadas en distintos países han estudiado la relación entre las habilidades sociales y la ansiedad social, y sus resultados indican que a menor nivel de competencia social se presenta mayor nivel de ansiedad tanto en poblaciones adolescentes (Navarro \& Yubero, 2012; Torrente, Piqueras, Orgilés \& Espada, 2014) como en estudiantes universitarios (Caballo, Salazar, Irurtia, Olivares \& Olivares, 2014). Por su parte, Fernández, Carranza y Ato (2012) corroboran los hallazgos de investigaciones previas al demostrar que el grado de ajuste emocional es un predictor del rendimiento y de las competencias académicas, así como sucede con variables como el coeficiente intelectual.
Del mismo modo, los niños que muestran un estilo caracterizado por la agresividad o la evitación pueden desarrollar problemas de ajuste psicosocial y tener un mayor riesgo de trastornos psicoafectivos, esto porque el déficit de habilidades sociales y asertividad dificulta hacer amigos y favorece el desarrollo de pautas de comportamiento agresivo, así como negativismo, transgresión, impulsividad, autoagresión e inhibición conductual; aspectos que pueden llevar a rechazo social, aislamiento, baja autoestima e indefensión (Lacunza, 2009).

Por otra parte, con respecto a las comparaciones por sexo, algunos estudios han reportado que las mujeres suelen mostrar más habilidades sociales que los hombres, particularmente en lo relativo a asertividad (García et al., 2014; Lacunza, 2009); que en adolescentes y jóvenes con pocas habilidades sociales, las mujeres presentan niveles más altos de ansiedad social que los hombres -tanto en lo relativo a sus componentes cognitivos como en sus reacciones fisiológicas-en tanto que estos tienen mayores conductas de evitación o escape (Inglés et al., 2010b; Zubeidat, Fernández, Sierra \& Salinas, 2008); y que los hombres tienen mayor tendencia a atribuir sus éxitos académicos a sus propias capacidades y muestran mayor seguridad personal, lo que representa un estilo atribucional más adaptativo y una mayor autoestima (Inglés et al., 2012).

Otros datos relacionados con poblaciones adolescentes son aportados por Contreras et al. (2005), pues, en el estudio realizado con una muestra de 120 estudiantes colombianos de secundaria encontraron que la ansiedad se correlacionaba con el rendimiento académico en las áreas de matemáticas y ciencias. Asimismo, el estudio de Lozano y García (2000) con una muestra de 684 estudiantes españoles de secundaria indicó que los alumnos de ambos sexos presentaban altos niveles de ansiedad y agresividad; acá, las mujeres mostraron lo que los autores denominan una patología de tipo interno, caracterizada por conductas depresivas, quejas somáticas y problemas de relación interpersonal -como agresividad verbal- en mayor medida que los hombres; mientras que estos presentaban con mayor frecuencia que las mujeres síndromes principalmente externos, como búsqueda de atención, desobediencia, conducta agresiva física y amenazas, que derivaban incluso en conducta delictiva.

En dicha muestra se observó correlación entre los niveles de agresividad y rendimiento escolar (calificaciones escolares), pero Cohen et al. (2011), en su estudio realizado con estudiantes argentinos, reportaron que las mujeres obtuvieron puntajes significativamente más altos en la escala de Ansiedad social/Timidez, lo que tiene grandes implicaciones porque, como reportan los autores, "la incompetencia social se vincula con bajo rendimiento, fracaso, ausentismo y expulsión de la escuela” (p. 169). 
Es importante mencionar que si bien es cierto que las investigaciones citadas han permitido avanzar en el estudio de las relaciones que guardan las habilidades sociales, la ansiedad y el rendimiento académico en adolescentes, no es posible generalizar sus resultados a poblaciones mexicanas. Además, la mayor parte de dichas investigaciones ha medido lo que se denomina ansiedad social, definida como un miedo intenso y persistente ante diversas situaciones sociales, donde la persona puede ser evaluada negativamente por otras. Pero, dadas las implicaciones que tiene la ansiedad en el funcionamiento psicológico general, y que incluso puede considerarse un indicador pertinente de la falta de ajuste emocional (Sánchez, Aparicio \& Dresch, 2006), reducir la evaluación únicamente a la ansiedad social compromete información que podría ser relevante. Específicamente, la ansiedad puede ser provocada tanto por estímulos tanto externos como internos, incluyendo pensamientos y expectativas; y altos niveles de ansiedad pueden derivar en manifestaciones fisiológicas (dificultades para dormir, sudoración, cansancio, entre otros) que pueden asociarse con errores psicomotores o intelectuales, dificultad para la concentración y alteración del funcionamiento psicológico, en general, en cualquier tarea que requiera atención, concentración y memoria (Alccalaico, 2012).

Por último, es relevante también mencionar el trabajo de Reynolds y Richmond (1997), quienes desarrollaron la Escala de Ansiedad Manifiesta (CMAS), concibiendo el constructo ansiedad de manera amplia y compleja, donde se incluyen diversos componentes, como la ansiedad fisiológica, la inquietud/hipersensibilidad y las preocupaciones sociales/concentración. Cada uno de esos aspectos puede estar relacionado con la presencia o ausencia de habilidades sociales como la asertividad, así como con su contraparte, la agresividad/pasividad. Por tales razones, para la presente investigación se consideró el uso de dicha escala.

Ante el panorama expuesto surge la necesidad de conocer la relación que guardan las variables descritas en población mexicana y resaltar su importancia e influencia en el rendimiento escolar. Así, el objetivo de la presente investigación fue conocer la relación entre el nivel de asertividad, el rendimiento académico y diversos componentes de la ansiedad en una muestra de estudiantes mexicanos de secundaria, así como explorar posibles diferencias por sexo. Con base en lo reportado por la literatura, se planteó como hipótesis de investigación que se encontraría una correlación entre las diversas variables a evaluar, es decir: a menor nivel de ansiedad, mayor asertividad y mayor rendimiento académico.

\section{MÉTODO}

\section{Tipo de estudio}

Para el presente trabajo se utilizó un diseño descriptivo y correlacional (Hernández, Fernández \& Baptista, 2014).

\section{Participantes}

La muestra estuvo conformada por 535 estudiantes de secundaria (54.6\% mujeres y $45.4 \%$ hombres), con un promedio de edad de 13.01 años $(D E=.74$, rango de 11 a 17 años), inscritos en siete grupos de primer grado y en seis grupos de segundo grado, en una escuela pública localizada en un municipio de Hidalgo, México. El muestreo fue por conglomerados y conveniencia, abarcando la totalidad de los alumnos de los dos primeros grados escolares del plantel. Esta evaluación es parte de la primera fase de una investigación más amplia, donde a partir de los resultados obtenidos se diseñará e instrumentará una intervención psicológica con los estudiantes evaluados que obtengan puntajes que indican algún tipo de riesgo.

\section{Instrumentos}

Para la medición de variables se utilizaron dos escalas:

Escala de Conducta Asertiva para Niños (CABS) (Michelson \& Wood, 1982). Específicamente, se utilizó la adaptación de Lara y Silva (2002), quienes estandarizaron la escala para población infantil y adolescente mexicana para un rango de edad de 9 a 17 años, y donde se obtuvo una consistencia interna de .80. Este instrumento está compuesto por 27 reactivos con cinco opciones de respuesta, divididos en: Factor 1: Dar y recibir cumplidos y críticas, con 20 reactivos diseñados para evaluar si el niño o adolescente se comporta de manera asertiva, pasiva o agresiva en su interacción con diversas personas y situaciones sociales; $\mathrm{y}$ Factor 2: Enojo y culpa, con siete reactivos que evalúan las reacciones ante situaciones que le son molestas. Los valores alfa de Cronbach obtenidos en la presente investigación fueron de: .74 para el Factor 1, .62 para el Factor 2 y .80 para la Escala Total de Asertividad.

Esta escala define la calificación de cada respuesta con un valor de 0 a 5 puntos para caracterizar, en la suma total de las puntuaciones, el comportamiento como asertivo (puntajes entre 27 y 42), pasivo (entre 43 y 50) o agresivo (entre 51 y 135). Donde el estilo asertivo se refiere a respetar los derechos propios y los de los demás; mientras que el estilo pasivo se caracteriza por conductas de indefensión, sometimiento y culpa que impiden defender los derechos propios e implican la aceptación de todo lo que 
otros pidan; y el estilo agresivo corresponde a conductas y actitudes que signifiquen agredir a los demás sin tomarlos en cuenta. Las calificaciones de la escala se interpretan de manera inversa, es decir: a menor puntaje, mayor habilidad social (asertividad), y a mayor puntaje, mayor agresividad.

Escala de ansiedad manifiesta en niños -revisada(CMAS-R) (Reynolds \& Richmond, 1997). Este instrumento fue diseñado para valorar el nivel y la naturaleza de la ansiedad en poblaciones de 6 a 19 años de edad. Específicamente, en esta investigación se utilizó la versión de Bautista (2000), quien obtuvo validez, confiabilidad y consistencia interna del instrumento con población mexicana, con un valor alfa de Cronbach de .74. El alfa de Cronbach obtenida en la presente investigación fue de .73 .

En total, la escala consta de 37 reactivos agrupados en cuatro subescalas: Ansiedad fisiológica, con 10 reactivos (la confiabilidad Kuder-Richardson obtenida en esta investigación fue de .63); Inquietud/hipersensibilidad, con 11 reactivos (confiabilidad K-R en esta investigación de .69); Preocupaciones sociales/concentración, con 10 reactivos (confiabilidad K-R en esta investigación de .40); y Mentira, con siete reactivos (confiabilidad K-R en esta investigación de .27). Cada reactivo se responde con un sí o un no, con asignación de valor de 0 y 1 . Las puntuaciones pueden variar de 0 a 28 , donde a mayor puntuación, mayor nivel de ansiedad. Los valores por debajo de 13 puntos se consideran dentro de los estándares normales de síntomas ansiosos que pueden experimentar los adolescentes, mientras que los valores de 13 a 28 puntos indican alto nivel de ansiedad, el cual puede considerarse de nivel clínico. La subescala de mentira no se considera en la puntuación total de la ansiedad, sino que es solo un indicativo de que el participante puede estar mintiendo en sus respuestas; en caso de que se encuentren valores altos de mentira, la prueba total se invalida para ese participante.

Finalmente, para obtener el nivel de rendimiento académico se consideró el promedio de calificaciones obtenidas por los alumnos en las materias español, matemáticas y ciencias naturales, al final del ciclo escolar. Cabe aclarar que en el sistema escolar mexicano se califica a los alumnos con una escala de 0 a 10 puntos, donde el mínimo aprobatorio es de 6, y el 10 indica excelencia.

\section{Procedimiento}

La aplicación de los instrumentos fue llevada a cabo por 15 estudiantes de licenciatura en psicología, quienes previamente recibieron capacitación por parte de los investigadores. En cada grupo escolar se presentaron cuatro aplicadores para solicitar la colaboración de los alumnos, darles las instrucciones y repartir los cuestionarios. Los alumnos respondieron individualmente los instrumentos; $\mathrm{y}$ cuando cada uno concluía, levantaba la mano, permaneciendo sentado, y uno de los aplicadores le recogía los formatos. El profesor de cada grupo estuvo presente durante los 50 minutos asignados para la sesión de aplicación de los instrumentos, pero no participó en el proceso de aplicación. Al concluir, se agradeció la participación de los alumnos.

\section{Análisis de datos}

Los instrumentos aplicados fueron calificados por uno de los investigadores del presente estudio. Todos los datos fueron capturados en una base de datos para su análisis con el Paquete Estadístico de Ciencias Sociales (SPSS v. 20). Con este, se obtuvieron los puntajes individuales, los promedios para cada instrumento y los promedios escolares. Asimismo, los datos fueron agrupados de acuerdo al sexo de los participantes para realizar la comparación de las medias a través de la prueba $t$ de Student. También, se obtuvo el coeficiente de correlación de Pearson para conocer la relación entre todas las variables evaluadas: (a) asertividad/agresividad, (b) ansiedad y (c) rendimiento académico (promedio escolar). Cabe aclarar que el tamaño de la muestra de la presente investigación $(>30)$ puede considerarse grande; y, de acuerdo al Teorema del Límite Central, en muestras grandes los datos tienden a la normalidad y se les considera representativos de la población (Field, 2009). Esto justifica el uso de las pruebas estadísticas aquí utilizadas.

\section{Consideraciones éticas}

La investigación fue autorizada por las instituciones escolares correspondientes. El director de la escuela, así como el Consejo Técnico escolar, aprobaron que se llevara a cabo la aplicación de los instrumentos un mes antes de concluir el ciclo escolar. Se aclaró a los alumnos que su participación era voluntaria y confidencial, y que podían dejar de responder en el momento que lo desearan, sin sanción alguna; sin embargo, ningún estudiante se rehusó a contestar los cuestionarios. Adicionalmente, las autoridades y profesores recibieron un informe de los resultados en el que se mantuvo el anonimato de los alumnos, informe que fue utilizado como base para elaborar un programa de intervención.

\section{RESULTADOS}

En esta sección se presentan los resultados de la comparación de las medias obtenidas en cada instrumento aplicado: Escala de Asertividad y Escala de Ansiedad, así como en Rendimiento Académico (promedio escolar); 
posteriormente, se presentan las correlaciones obtenidas entre los valores de los instrumentos y el promedio escolar.

En la Tabla 1 se presentan-de manera comparativa-los hallazgos con respecto a la obtención de medias para los puntajes de los hombres y de las mujeres participantes en lo relativo al promedio escolar, el total de cada instrumento aplicado y en cada subescala.

Según encontrado en la Escala de Asertividad, los puntajes entre 51 y 135 puntos indican patrones caracterizados por déficits de habilidades sociales y presencia de conducta agresiva. Específicamente, los puntajes promedio obtenidos por los hombres $(M=55.19, D E=12.73)$ fueron mayores a los obtenidos por las mujeres $(M=52.73, D E=12.95)$, y el análisis indicó diferencias estadísticamente significativas $(t=$ $-2.19, p<.05)$. Asimismo, la comparación por subescalas arrojó diferencias significativas en el factor 1 -Dar y recibir cumplidos y críticas $-(t=-2.49, p<.05)$, lo que indica que los hombres muestran menor asertividad en ese aspecto, y tienden a comportarse de manera más agresiva que las mujeres al recibir una crítica, al expresar sus sentimientos, al iniciar una conversación o al hacer una petición. Por otro lado, no se encontraron diferencias por sexo en lo relativo al factor 2 -Enojo y culpa-, porque tanto hombres como mujeres reaccionan de forma predominantemente agresiva ante situaciones que les son molestas.

Por otra parte, los hombres y las mujeres obtuvieron puntajes promedio que caen dentro de los estándares normales de ansiedad $(M=10.93, D E=4.88 ; M=12.50$, $D E=5.16$, respectivamente), sin embargo, el promedio obtenido por ellas se ubica en el límite superior, cercano al nivel clínico de ansiedad (13 puntos, según la escala). Además, las diferencias por sexo fueron estadísticamente significativas $(t=3.59, p<.01)$ y la comparación también indicó diferencias significativas en dos subescalas: en lo relativo a Ansiedad fisiológica, las mujeres reportaron mayores manifestaciones de ansiedad, incluyendo dificultades del sueño, náuseas, sudoración y fatiga; en Inquietud/ hipersensibilidad, en estas mismas se detectaron, en mayor medida que en los hombres, preocupaciones obsesivas (por ejemplo, estar pensando constantemente en los juicios que otros hagan de su conducta), así como miedo a ser lastimadas o aisladas emocionalmente, y a que les suceda algo malo; mientras que en Preocupaciones sociales/concentración no se encontraron diferencias significativas entre hombres y mujeres, donde, en ambos casos, los niveles obtenidos pueden considerarse como normales.

Respecto a la variable de Rendimiento Académico, los valores promedio pueden ubicarse en un nivel adecuado, siendo las mujeres quienes obtuvieron un mayor promedio escolar $(M=8.54, D E=.86)$, en comparación con los hombres $(M=8.03, D E=.85)$, diferencias que fueron estadísticamente significativas $(t=6.79, p<.01)$.

Por otro lado, en la Tabla 2 se puede observar que el total de la Escala de Asertividad, así como sus dos factores (Dar y recibir cumplidos y críticas, y Enojo y culpa), correlacionaron positivamente con el Total en la Escala de Ansiedad y con dos de sus subescalas (Ansiedad fisiológica y Preocupaciones sociales/concentración). Debe recordarse que, en la primera escala, los puntajes bajos indican que la persona se comporta asertivamente, mientras que los puntajes altos indican un patrón de conducta agresiva. Las correlaciones positivas en los dos indicadores mencionados implican que los jóvenes que se comportan agresivamente tienen mayores niveles de ansiedad.

Tabla 1

Comparación de las medias obtenidas en cada instrumento aplicado: Escala de Asertividad y Escala de Ansiedad, y en Rendimiento Académico (promedio escolar).

\begin{tabular}{|c|c|c|c|c|c|}
\hline \multirow{2}{*}{ Variables } & \multicolumn{2}{|c|}{ Hombres } & \multicolumn{2}{|c|}{ Mujeres } & \multirow[b]{2}{*}{$\mathrm{t}$} \\
\hline & M & $\mathrm{DE}$ & M & $\mathrm{DE}$ & \\
\hline TOTAL EN LA ESCALA DE ASERTIVIDAD & 55.19 & 12.73 & 52.73 & 12.95 & $-2.19 *$ \\
\hline Dar y recibir cumplidos y críticas & 36.84 & 8.89 & 34.96 & 8.56 & $-2.49 *$ \\
\hline Enojo y culpa & 18.13 & 5.43 & 17.77 & 5.38 & -1.20 \\
\hline $\begin{array}{l}\text { RENDIMIENTO ACADÉMICO } \\
\text { (Promedio escolar) }\end{array}$ & 8.03 & .85 & 8.54 & .86 & $6.79 * *$ \\
\hline TOTAL EN LA ESCALA DE ANSIEDAD & 10.93 & 4.88 & 12.50 & 5.16 & $3.59 * *$ \\
\hline Ansiedad fisiológica & 3.31 & 2.05 & 4.00 & 2.29 & $3.60 * *$ \\
\hline Inquietud/hipersensibilidad & 5.32 & 2.56 & 6.4 & 2.41 & $3.31 * *$ \\
\hline Preocupaciones sociales/concentración & 2.28 & 1.39 & 2.45 & 1.65 & 1.27 \\
\hline
\end{tabular}

Nota. ${ }^{*} p<.05,{ }^{* *} p<.01$. 
Tabla 2

Correlaciones entre los valores de la Escala de Asertividad, la Escala de Ansiedad, y el Rendimiento académico (promedio escolar).

\begin{tabular}{|c|c|c|c|c|c|c|c|c|c|}
\hline & Variables & 1 & 2 & 3 & 4 & 5 & 6 & 7 & 8 \\
\hline 1 & TOTAL EN ESCALA DE ASERTIVIDAD & - & $.946^{* *}$ & $.852 * *$ & $-.203 * *$ & $.182 * *$ & $.236 * *$ & .013 & $.240 * *$ \\
\hline 2 & Factor 1. Dar y recibir cumplidos y críticas & & -- & $.638^{* *}$ & $-.222 * *$ & $.157^{* *}$ & $.189 * *$ & .001 & $.244 * *$ \\
\hline 3 & Factor 2. Enojo y culpa & & & -- & $-.124 * *$ & $.180^{* *}$ & $.257 * *$ & .030 & $.177 * *$ \\
\hline 4 & $\begin{array}{l}\text { RENDIMIENTO ACADÉMICO } \\
\text { (Promedio escolar) }\end{array}$ & & & & -- & -.038 & -.072 & .062 & $-.124 * *$ \\
\hline 5 & TOTAL EN ESCALA DE ANSIEDAD & & & & & -- & $.850 * *$ & $.852 * *$ & $.695^{* *}$ \\
\hline 6 & Ansiedad fisiológica & & & & & & -- & $.557^{* *}$ & $.464^{* *}$ \\
\hline 7 & Inquietud/hipersensibilidad & & & & & & & -- & $.385^{* *}$ \\
\hline 8 & Preocupaciones sociales/concentración & & & & & & & & -- \\
\hline
\end{tabular}

Nota. ** Correlación significante al nivel .01. * Correlación significante al nivel .05.

También, se observa que la variable Rendimiento Académico (promedio escolar) correlacionó negativamente con el total de la Escala de Asertividad y con sus dos factores, aunque el grado de asociación fue bajo. Nuevamente, considerando la interpretación de los puntajes de esta escala, las correlaciones negativas encontradas implican que los jóvenes que se comportan agresivamente tienen menor rendimiento académico. Respecto a la correlación negativa encontrada con una de las subescalas de Ansiedad, se interpreta que a menor grado de preocupaciones sociales o problemas de concentración, los alumnos tienen mayor rendimiento académico.

Cabe aclarar que, aun cuando el análisis de correlaciones indicó que diversas variables se relacionaron de manera estadísticamente significativa, estas correlaciones pueden atribuirse al tamaño de la muestra. Además, algunas de dichas correlaciones fueron de baja magnitud (inferiores a .40).

\section{DISCUSIÓN}

De acuerdo con los resultados obtenidos, los objetivos de la investigación fueron cubiertos en su totalidad, aun cuando la hipótesis de investigación se cumplió solo parcialmente, dado que se encontraron correlaciones entre algunas, pero no entre todas las variables evaluadas.

Específicamente, la conducta de hombres y mujeres se puede ubicar dentro del tipo de interacción agresivo y con bajos niveles de asertividad, es decir, puede hablarse de un patrón de incompetencia social. Estos datos coinciden con lo reportado para adolescentes argentinos (Lacunza, 2009; Cohen et al., 2011), colombianos (Contreras et al., 2005; Oyarzún et al., 2012) y españoles (Gutiérrez \& López, 2015; Mendo et al., 2016; Redondo et al., 2014). Asimismo, en los participantes de la presente investigación, los hombres reportaron mayores niveles de agresividad que las mujeres. Dicha diferencia podría explicarse por los estereotipos de género que aún persisten en amplios sectores de la sociedad mexicana, pues, por tradición cultural, a muchos hombres mexicanos se les alienta a enfocarse al logro de metas y a la competitividad agresiva, así como a comportarse con rudeza; conductas que se consideran típicamente como masculinas. Incluso, los hombres pueden, ser castigados socialmente por expresar sentimientos positivos y, como lo señalan Ojeda, Melby, Sánchez y Rodarte (2007), aun cuando la expresión de sentimientos puede favorecer algunas relaciones interpersonales, en muchos casos se considera una de las características de la feminidad.

Por otro lado, el hecho de no encontrar diferencias por sexo entre el nivel de agresividad ante situaciones de enojo concuerda con lo reportado por Alcázar, Jurado y Reyes (2015), quienes explican que hombres y mujeres muestran una tendencia similar al enojo y a la expresión del mismo. Aunque aclaran que la forma de manifestación sí es distinta, pues mientras los hombres lo expresan físicamente con conductas como retar a golpes y golpear la pared, las mujeres recurren a aventar objetos o azotar la puerta. De cualquier modo, los resultados indican que los adolescentes de este estudio no han desarrollado un patrón de interacción social que les permita su integración adecuada al ambiente escolar. 
Con respecto a los niveles de ansiedad, el detectado en las mujeres fue muy cercano al nivel clínico, es decir, aquél que ya requiere atención psicológica especializada. Este resultado es consistente con estudios realizados en otros países (Inglés et al., 2010b; Zubeidat et al., 2008), los cuales reportan que las mujeres tienen mayor tendencia a la ansiedad que los hombres. Al respecto, es importante señalar que la ansiedad es la manera en que las personas responden a situaciones percibidas como amenazantes. Adicionalmente, según Carreño, Morales, Sánchez, Henales y Espíndola (2007), las conductas ansiosas son producto de disposiciones conductuales adquiridas que involucran residuos de experiencias pasadas y que predisponen al individuo a percibir al mundo como potencialmente peligroso; de este modo, las personas aprenden a responder con manifestaciones de ansiedad cuando no tienen estrategias adaptativas.

La explicación que puede darse a los mayores niveles de ansiedad en las mujeres se relaciona con aspectos culturales y de crianza, que son producto de los estereotipos y roles de género. Tradicionalmente, a las mujeres se les atribuyen rasgos como sensibilidad, afectividad, recato, vida doméstica y flexibilidad, que socialmente se consideran características femeninas; en concordancia con ello, muchas niñas son criadas en un ambiente que les limita salir de casa y pertenecer a diversos grupos sociales, y que también les exige el aprendizaje de labores domésticas, vestirse recatadamente, expresarse con respeto y propiedad (ante los adultos y personas de su propia edad), e, incluso, servir a los demás para ser coherentes con la conducta y forma de vida de las mujeres. Además, desde edades tempranas, a muchas mexicanas se les advierte de los peligros a los que están expuestas en el mundo, se les da un trato diferencial, se les sobreprotege y se les convence de su debilidad y necesidad de protección.

Todo lo anterior puede generar en las adolescentes una serie de miedos y preocupaciones obsesivas, que incluyen el estar pensando constantemente en los juicios que otros hacen de su conducta, tener excesivas inquietudes por su seguridad e integridad, así como miedo a ser lastimadas, a ser aisladas emocionalmente, a sentirse solas y a que les suceda algo; incluso, les puede generar manifestaciones de ansiedad fisiológica, expresadas en síntomas como cansancio, sudoración de manos, pesadillas, dificultades para dormir, irritabilidad, sensación de ahogo y náuseas, además de dificultad para concentrarse en tareas escolares. Características que fueron detectadas en las participantes del presente estudio.

Además, es necesario considerar que en la sociedad mexicana aún están presentes muchas prácticas parentales de control psicológico referentes a la manipulación del pensamiento y las emociones de niños y adolescentes (particularmente del sexo femenino), y que involucran crítica negativa, retiro de afecto, chantaje, comunicación restrictiva, desvalorización, sobreprotección y prácticas intrusivas (González \& Morales, 2016). Esto plantea la posibilidad de que la ansiedad aprendida, es decir, la serie de pensamientos que muchas mujeres adoptan como suyos e internalizan, tiendan a volverse hábitos de pensamiento que son desarrollados como cualquier hábito de conducta (Cartagena, 2008) y que pueden perdurar a lo largo de sus vidas.

El panorama descrito lleva necesariamente a la falta de habilidades sociales y asertividad, así como a problemas emocionales en muchos adolescentes mexicanos tanto hombres como mujeres, como se encontró en el presente estudio, aunque cabe resaltar que el promedio académico obtenido no parece constituir un problema, al ser superior de 8 de 10, en ambos casos.

Al tomar en consideración los datos de toda la muestra estudiada, los hallazgos indican una relación entre niveles de asertividad y de ansiedad. Dado que los bajos niveles de asertividad implican alto grado de agresividad -conductas caracterizadas por peleas, acusaciones, amenazas, y, en general, por actitudes que signifiquen agredir a los demás sin tomarlos en cuenta-, cabe decir que dichos aspectos se asociaron con un mayor nivel de síntomas ansiosos. Estos resultados concuerdan con los hallazgos de diversas investigaciones (Caballo et al., 2014; Cohen et al., 2011; Inglés et al., 2010a; Navarro \& Yubero, 2012; Torrente et al., 2014), que coinciden en que, a mayor déficit en habilidades sociales o asertividad -que se manifiesta en conductas agresivas-, el número de síntomas ansiosos es mayor. Relación que se presenta, de manera consistente en diferentes etapas del desarrollo.

Es interesante notar que ninguno de los factores de la Escala de Asertividad correlacionó con la subescala de Inquietud/hipersensibilidad de la Escala de Ansiedad, la cual está asociada con la preocupación obsesiva por ideas indefinidas. Ese último hallazgo sugiere que el déficit en las habilidades sociales sí está asociado con la ansiedad, pero no necesariamente con todas sus áreas de manifestación. $\mathrm{Y}$ es importante señalar que algunos adolescentes pueden haber desarrollado habilidades sociales, pero ante la presencia de elevados niveles de ansiedad, tales habilidades pueden inhibirse.

Por otro lado, los hallazgos del presente estudio también indicaron una relación entre habilidad social, ansiedad y rendimiento académico. Lo que confirma que los alumnos con bajo desempeño en asertividad, altos niveles de agresividad y altos niveles de ansiedad son también los que muestran menor rendimiento académico. Al respecto, cabe 
agregar los señalamientos de Contreras et al. (2005), en el sentido de que la ansiedad leve o moderada puede cumplir una función útil o favorecedora para al individuo, porque genera un estado de alerta y de tensión que puede mejorar el rendimiento académico; sin embargo, altos niveles de ansiedad pueden conducir a errores en el desempeño de tareas intelectuales debido a que resultan afectados los procesos de memoria, concentración y funcionamiento psicológico del estudiante.

Aun cuando el promedio académico ubicado en el presente estudio no puede considerarse bajo, los hallazgos en general resultan preocupantes dado que los adolescentes corren riesgo de desarrollar problemas de ajuste psicosocial, trastornos psicoafectivos, rechazo social, aislamiento y baja autoestima ante sus deficientes niveles de habilidad social y asertividad (Castro, 2005; Gutiérrez \& López, 2015; Inglés et al., 2010a, 2012); además, particularmente en el caso de las mujeres, se corren también los riesgos relacionados con la ansiedad antes mencionados.

Dado lo anterior, se hace necesario desarrollar programas de prevención e intervención basados en la enseñanza explícita de habilidades sociales -especialmente de asertividad- en las aulas de nivel primaria y secundaria. Esto es de gran importancia dado que la capacidad de los niños y adolescentes para relacionarse con otras personas asertivamente contribuye en todos los aspectos de su desarrollo (Bermúdez, 2010) y tiene un fuerte impacto en la organización psicológica del individuo, en su calidad de vida y en la inclusión social (Cohen et al., 2011).

Cabe mencionar que investigaciones previas han probado la efectividad de los programas de intervención en habilidades sociales para reducir los niveles de ansiedad (Monjas, 2012; Olivares, Olivares \& Macià, 2014; Wagner, Pereira \& Oliveira, 2014), y dichos programas enfatizan la importancia de que los adolescentes aprendan a relacionarse con sus pares y profesores en el aula y en otros ambientes escolares y sociales. Esto pone de manifiesto la importancia de integrar al currículum educativo, como parte esencial de la escolarización, programas dirigidos al desarrollo de habilidades sociales que enseñen explícitamente habilidades para relacionarse asertivamente. Incrementar la competencia social dentro del aula ayudaría a mejorar la integración del adolescente con sus pares, a disminuir la agresividad y a que desarrollaran comportamientos más adaptativos (Castro, 2005).

También, el fracaso social en la escuela, manifestado a través del rechazo o la agresividad, es un factor de riesgo para el desarrollo integral del niño y adolescente, y, por tanto, puede llevar a un rendimiento escolar bajo que, eventualmente, podría derivar en fracaso escolar. Como señalan Contreras et al. (2005), el fracaso escolar "constituye un problema grave a nivel humano, moral y social, que a menudo genera exclusiones que marcarán a los jóvenes durante toda su vida" (p. 187).

Dentro de las limitaciones del estudio se puede señalar la baja confiabilidad de las subescalas Preocupaciones sociales/ Concentración (.40) y Mentira (.27), que forman parte de la escala de Ansiedad. Si bien el Total de Ansiedad tuvo una confiabilidad aceptable, esta disminuyó ligeramente al incorporar los datos de la primera subescala mencionada. Respecto a la subescala de Mentira, aun cuando no es considerada para el puntaje del Total de Ansiedad, el hecho de obtener baja confiabilidad limita el grado de consistencia de los datos. Lo anterior sugiere la pertinencia de que, en futuros estudios, se busquen otras opciones con respecto a los instrumentos, o bien, se incorporen nuevos reactivos a las subescalas, lo que permitirá contar con un mayor grado de consistencia de los hallazgos.

Para concluir, es importante señalar la necesidad de incorporar en el análisis la inclusión de otros constructos. Es conveniente evaluar en los alumnos otros indicadores psicológicos tanto de bienestar como de desajuste psicológico, por ejemplo, el nivel de autoestima, autoeficacia y motivación, que se relacionan tanto con las habilidades sociales como con el rendimiento académico. Investigaciones como la realizada por Becerra y Reidl (2015) con estudiantes mexicanos de bachillerato indican que el rendimiento académico se correlaciona con el estilo atribucional, la motivación de logro y la autoeficacia. Las intervenciones orientadas al desarrollo de la competencia social, así como a la promoción de factores protectores que coadyuven a mejorar el rendimiento académico y el ajuste social en los adolescentes, han de abarcar diversos aspectos de la problemática, adaptarse al contexto escolar y dirigirse a promover un desarrollo integral de los alumnos en el contexto escolar.

\section{REFERENCIAS}

Alcázar, R., Jurado, S., \& Reyes, V. (2015). El enojo como rasgo de personalidad y agresión física en adolescentes de preparatoria. PSICUMEX, 5(1), 89-101. Recuperado de http:// www.psicumex.uson.mx/revistas/articulos/9-R9_ARTICULO_06.pdf.

Alccalaico, M. (2012). Características del trastorno de ansiedad o fobia social en el adolescente. Revista Cientifica de Ciencias de la Salud, 5(5), 61-68. Recuperado de http:// revistascientificas.upeu.edu.pe/index.php/rc_salud/article/ view/179/700.

Bandura, A. (1982). Teoría del aprendizaje social. Madrid: Espasa Calpe.

Bautista, G. (2000). Validez y confiabilidad de la escala de ansiedad manifiesta en niños y adolescentes mexicanos (Tesis 
de Especialidad en Psiquiatría, no publicada). Facultad de Medicina. Universidad Nacional Autónoma de México.

Becerra, C., \& Reidl, L. (2015). Motivación, autoeficacia, estilo atribucional y rendimiento escolar en estudiantes de bachillerato. Revista Electrónica de Investigación Educativa, 17(3), 79-93. Recuperado de http://www.scielo.org.mx/pdf/ redie/v17n3/v17n3a6.pdf.

Bermúdez, M. (2010). Diseño, construcción y análisis psicométrico de una escala de competencia social para niños de 3 a 6 años versión padres de familia. Revista Iberoamericana de Psicología: Ciencia y Tecnología, 3(1) 49-65. Recuperado de http://revistas.iberoamericana.edu.co/index.php/ripsicologia/article/view/192.

Caballo, V. (1993). Manual de evaluación y entrenamiento de las habilidades sociales. Madrid: Siglo XXI.

Caballo, V. (2009). Manual de técnicas de terapia y modificación de conducta. México: Siglo XXI.

Caballo, V., Salazar, I., Irurtia, M., Olivares, P., \& Olivares, J. (2014). Relación de las habilidades sociales con la ansiedad social y los estilos/trastornos de la personalidad. Psicología Conductual, 22 (3), 401-422. Recuperado de https://dialnet. unirioja.es/servlet/articulo?codigo $=4916443$.

Carbonero, M., Martín, L., Monsalvo, E., \& Valdivieso, J. (2015). Rendimiento escolar y actitudes personales y de responsabilidad social en el alumnado preadolescente. Anales de Psicología, 31(3), 990-999. doi:10.6018/analesps.31.3.181161.

Carreño, J., Morales, F., Sánchez, C., Henales, C., \& Espíndola, J. (2007). Una explicación de los síntomas de depresión y ansiedad en mujeres estériles. Ginecología y Obstetricia de México, 75(3), 133-141. Recuperado de http://new.medigraphic.com/cgi-bin/resumen.cgi?IDARTICULO=17903.

Cartagena, M. (2008). Relación entre la autoeficacia y el rendimiento escolar y los hábitos de estudio en alumnos de secundaria. Revista Iberoamericana sobre Calidad, Eficacia y Cambio en Educación, 6(3), 60-89. Recuperado de http:// www.redalyc.org/pdf/551/55160304.pdf.

Castro, A. (2005). Alfabetización emocional: la deuda de enseñar a vivir con los demás. Revista Iberoamericana de Educación, 37(6), 2-15. Recuperado de https://dialnet.unirioja. es/servlet/articulo?codigo $=3197631$.

Cohen, S., Esterkind, E., Lacunza, A., Caballero, V., \& Martinenghi, C. (2011). Habilidades sociales y contexto sociocultural. Un estudio con adolescentes a través del BAS-3. Revista Iberoamericana de Evaluación Psicológica, 29(1), 167-183. Recuperado de https://dialnet.unirioja.es/servlet/ articulo? codigo $=5064348$.

Contreras, F., Espinosa, J., Esguerra, G., Haikal, A., Polanía, A., \& Rodríguez, A. (2005). Autoeficacia, ansiedad y rendimiento académico en adolescentes. Perspectivas en Psi- cología, 1(2), 183-194. Recuperado de http://www.redalyc. org/articulo.oa? id=67910207.

Costa, S., \& Tabernero, C. (2012). Rendimiento académico $\mathrm{y}$ auto-concepto en estudiantes de Educación Secundaria Obligatoria, según el género. Revista Iberoamericana de Psicología y Salud, 3(2), 175-193. Recuperado de http:// www.redalyc.org/articulo.oa?id=245124456006.

Fernández, C., Carranza, J., \& Ato, M. (2012). Efecto del ajuste socio-emocional en el rendimiento y las competencias académicas en el contexto escolar: Estudio comparativo. Anales de Psicología, 28(3), 892-903. doi:10.6018/analesps.28.3.131211.

Field, A. (2009). Discovering statistics using SPSS. (Third Ed.). España: Sage.

García, M., Cabanillas, G., Morán, V., \& Olaz, F. (2014). Diferencias de género en habilidades sociales en estudiantes universitarios de Argentina. Anuario Electrónico de Estudios en Comunicación Social, 7(2), 114-134. Recuperado de https://revistas.urosario.edu.co/index.php/disertaciones/ article/view/3736.

González, D., \& Morales, J. (2016). Relación entre las prácticas parentales, los estilos de aprendizaje y la masculinidadfeminidad en estudiantes de bachillerato. (Tesis de licenciatura no publicada). Universidad Autónoma del Estado de Hidalgo. Instituto Ciencias de la Salud. Área Académica de Psicología. México.

Gutiérrez, M., Escartí, A., \& Pascual, C. (2011). Relaciones entre empatía, conducta prosocial, agresividad, auto-eficacia y responsabilidad personal y social de los escolares. Psicothema, 23(1), 13-19. Recuperado de http://www.psicothema.com/pdf/3843.pdf.

Gutiérrez, M., \& López, J. (2015). Auto-concepto, dificultades interpersonales, habilidades sociales y conductas asertivas en adolescentes. Revista Española de Orientación y Psicopedagogía, 26(2), 42-58. Recuperado de http://www.redalyc.org/articulo.oa?id=338241632004.

Hernández, R., Fernández, C., \& Baptista, P. (2014). Metodología de la investigación. México: McGraw-Hill.

Inglés, C., Delgado, B., García, J., Ruiz, C., \& Díaz, Á. (2010a). Sociometric types and social interaction styles in a simple of spanish adolescents. The Spanish Journal of Psychology, 13(2), 730-740. Recuperado de http://www.redalyc.org/ pdf/172/17217376019.pdf.

Inglés, C. J., Díaz, A., García, J., Ruiz, C., Delgado, B., \& Martínez, M. (2012). Auto-atribuciones: diferencias de género y curso en estudiantes de Educación Secundaria. Revista Latinoamericana de Psicología, 44(3), 65-82. Recuperado de http://www.redalyc.org/articulo.oa?id=80525022005.

Inglés, C., Piqueras, J., García, J., García, L., Delgado, B., \& Ruiz, C. (2010b). Diferencias de género y edad en respuestas cognitivas, psicofisiológicas y motoras de ansie- 
dad social en la adolescencia. Psicothema, 22(3) 376-381. Recuperado de http://www.psicothema.com/psicothema. asp? $\mathrm{id}=3740$.

Lacunza, A. (2009). Las habilidades sociales como recurso para el desarrollo de fortalezas de la infancia. Psicodebate. Psicología, Cultura y Sociedad (España), X, 231-245. Recuperado de http:/www.palermo.edu/cienciassociales/ publicaciones/pdf/psico10/10Psico_13.pdf.

Lara, M., \& Silva, A. (2002) Estandarización de la Escala de Asertividad de Michelson y Wood en niños y adolescentes: II. (Tesis de Licenciatura, no publicada). Universidad Nacional Autónoma de México. Facultad de Psicología. México.

Lozano, L., \& García, E. (2000). El rendimiento escolar y los trastornos emocionales y comportamentales. Psicothema, 12(supl.2), 340-343. Recuperado de http://www.redalyc. org/articulo.oa?id=72797079.

Mendo, S., León, B., Felipe, E., Polo, M., \& Palacios, V. (2016). Evaluación de las habilidades sociales en estudiantes de Educación Social. Revista de Psicodidáctica, 21(1), 139-156. Recuperado de http:/www.ehu.eus/ojs/index.php/ psicodidactica/article/view/14031/13525.

Michelson, L., \& Wood, R. (1982). Development and psychometric properties of the children's assertive behavior scale CABS. Journal of Behavioral Assessment, 4(1), 3-13. Recuperado de http://link.springer.com/ article/10.1007\%2FBF01321378.

Monjas, I. (2012). Programa de enseñanza de habilidades de interacción social (PEHIS). Madrid: Ciencia de la educación preescolar y especial.

Navarro, R., \& Yubero, S. (2012). Impacto de la ansiedad social, las habilidades sociales y la cibervictimización en la comunicación online. Escritos de Psicología, 5(3), 4-15. Recuperado de https://investigacion.uclm.es/documentos/ fi_1357635991-vol5num32.pdf.

Ojeda, A., Melby, L., Sánchez, V., \& Rodarte, M. (2007). Correlación entre rasgos de masculinidad-feminidad y satisfacción marital en migrantes mexicanos. Migración y desarrollo, 8, 48-65. Recuperado de http://www.redalyc.org/ pdf/660/66000803.pdf.

Olivares, J., Olivares, P., \& Macià, D. (2014). Entrenamiento en habilidades sociales y tratamiento de adolescentes con fobia social generalizada. Psicología Conductual, 22(3),
441-459. Recuperado de https://dialnet.unirioja.es/servlet/ articulo? codigo $=4916449$.

Oyarzún, G., Estrada, C., Pino, E., \& Oyarzún, M. (2012). Habilidades sociales y rendimiento académico: Una mirada desde el género. Acta Colombiana de Psicología, 15(2), 21-28. Recuperado de http://repository.ucatolica.edu.co/ handle/10983/961.

Papalia, D., Olds, S., \& Feldman, R. (2009). Psicología del desarrollo. De la infancia a la adolescencia. 11'. edición. México: McGraw Hill.

Redondo, J., Inglés, C., \& García, J. (2014). Conducta prosocial $\mathrm{y}$ atribución académica en Educación Secundaria Obligatoria. Anales de Psicología, 30(2), 482-489. doi: 10.6018/ analesps.30.2.148331

Reynolds, C., \& Richmond, B. (1997). Escala de Ansiedad Manifiesta en Niños (Revisada). Manual Moderno: México.

Ribes, R., Bisquerra, R., Agulló, M., Fililla, G., \& Soldevila, A. (2005). Una propuesta de currículo emocional en educación infantil (3-6 años). Cultura y Educación, 17(1), 5-17.

Sánchez, M., Aparicio, M., \& Dresch, V. (2006). Ansiedad, autoestima y satisfacción autopercibida como predictores de la salud: diferencias entre hombres y mujeres. Psicothema, 18(3), 584-590. Recuperado de http://www.unioviedo.es/ reunido/index.php/PST/article/view/8476.

Torrente, E., Piqueras, J., Orgilés, M., \& Espada, J. (2014). Asociación de la adicción a Internet con la ansiedad social y la falta de habilidades sociales en adolescentes españoles. Terapia psicológica, 32(3), 175-184. Recuperado de http:// www.scielo.cl/pdf/terpsicol/v32n3/art01.pdf.

Wagner, M., Pereira, A., \& Oliveira, M. (2014). Intervención sobre las dimensiones de la ansiedad social por medio de un programa de entrenamiento en habilidades sociales. Psicología Conductual, 22(3), 423-440. Recuperado de https:// dialnet.unirioja.es/servlet/articulo?codigo $=4916445$.

Zubeidat, I., Fernández, A., Sierra, J., \& Salinas, J. (2008). Evaluación de factores asociados a la ansiedad social y a otras psicopatologías en adolescentes. Salud Mental, 31, 189-196. Recuperado de http://www.redalyc.org/pdf/582/58231304. pdf. 